\title{
Safety, Tolerability, Pharmacokinetics, and Pharmacodynamics of Atacicept in a Randomized Trial in Healthy Caucasian and Japanese Subjects
}

\author{
Daniela Willen ${ }^{1} \cdot$ Wolfgang Uhl $^{1} \cdot$ Peter Wolna ${ }^{1} \cdot$ Orestis Papasouliotis ${ }^{2} \cdot$ Özkan Yalkinoglu $^{1}$ (1)
}

Published online: 16 September 2019

(c) The Author(s) 2019

\begin{abstract}
Background and Objective Atacicept is an inhibitor of the B lymphocyte stimulator (BLyS) and a proliferation-inducing ligand (APRIL), and is being studied in relation to immunological disease. Currently, limited data on atacicept are available in non-Caucasian subjects. Pharmacokinetic data from earlier studies of atacicept were derived using an enzyme-linked immunosorbent assay (ELISA), which was subsequently found to have inadequacies. Hence, a new bioanalytical ELISA for total atacicept was developed and validated. We conducted this randomized, double-blind, placebo-controlled phase I study to compare the safety, tolerability, pharmacokinetics, and pharmacodynamics of atacicept in healthy Japanese and Caucasian subjects while generating pharmacokinetic data using the new ELISA.

Methods Japanese subjects aged $\geq 18$ to $\leq 55$ years $(n=24)$ were randomized (1:1:1:1) to a single subcutaneous dose of atacicept 25,75 , or $150 \mathrm{mg}$ or placebo. Caucasian subjects were then enrolled to match the Japanese subjects' gender, body weight $( \pm 20 \%)$, and height $( \pm 15 \%)$.

Results Atacicept was well tolerated and there were no clinically significant differences in treatment-emergent adverse events (TEAEs), vital signs, or laboratory parameters between the Japanese and Caucasian subjects. Most (90\%) TEAEs were mild; no severe or serious TEAEs or deaths occurred. Weight-adjusted atacicept exposure was comparable between ethnicities and across doses: the Japanese/Caucasian ratio of the area under the serum concentration-time curve from time zero to the last sampling point $\left(\mathrm{AUC}_{0-t}\right)$ was $107.21 \%(90 \% \mathrm{CI} 93.42-123.02 \%)$ and the Japanese/Caucasian ratio of maximum serum concentration $\left(C_{\max }\right)$ was $95.74 \%$ (90\% CI 74.26-123.43\%; ANCOVA). Median time to reach $C_{\max }\left(t_{\max }\right)$ was $20-60 \mathrm{~h}$ across all subjects. Dose-exposure relationships were comparable for the two ethnicities, with dose-normalized $\mathrm{AUC}_{0-t}$ decreasing with increasing dose, indicating nonlinear pharmacokinetics for the doses examined. There were no statistically significant differences between ethnicities in the pharmacokinetics-dose relationship. Some transient dose-related decreases in mean serum immunoglobulin (Ig)A and IgM, but not IgG, were observed after atacicept administration. There were small transient increases in peripheral B cell numbers in the first 4 days after dosing that were larger with atacicept than with placebo, with no apparent dose relationship. No anti-atacicept antibodies were detected.

Conclusion The safety, pharmacokinetic, and pharmacodynamic profiles of atacicept in healthy Japanese subjects were comparable to those in healthy Caucasian subjects.
\end{abstract}

EudraCT-ID: 2013-002703-34.

Electronic supplementary material The online version of this article (https://doi.org/10.1007/s13318-019-00575-7) contains supplementary material, which is available to authorized users.

Özkan Yalkinoglu

oezkan.yalkinoglu@merckgroup.com

1 Merck KGaA, Frankfurter Str. 250, 64293 Darmstadt, Germany

2 Merck Institute for Pharmacometrics (An Affiliate of Merck KGaA, Darmstadt, Germany), Lausanne, Switzerland

\section{Introduction}

Atacicept is a recombinant fusion protein comprising the extracellular ligand-binding domain of the transmembrane activator and calcium-modulator and cyclophilin-ligand interactor (TACI) receptor fused to a modified $\mathrm{Fc}$ portion of human immunoglobulin (Ig) G1 [1].

Atacicept acts as a dual antagonist of the homo- and heterotrimeric forms of two B-cell-stimulating cytokines, the B lymphocyte stimulator [BLyS; also known as BAFF 


\section{Key Points}

Atacicept is a dual inhibitor of B lymphocyte stimulator (BLyS) and a proliferation-inducing ligand (APRIL), and is in clinical development for the treatment of systemic lupus erythematosus. Thus far, the pharmacokinetics and pharmacodynamics of atacicept have predominantly been investigated in Caucasian subjects.

In this study, we assessed atacicept safety, tolerability, pharmacokinetics, and pharmacodynamics in healthy Japanese and Caucasian volunteers and found that: (a) safety outcomes were similar for the two ethnicities; (b) weight-adjusted atacicept exposure parameters were comparable in Japanese and Caucasian subjects, with a less than dose-proportional increase in the area under the serum concentration-time curve from time zero to the last sampling point $\left(\mathrm{AUC}_{0-t}\right)$ with increasing dose; $(\mathrm{c})$ pharmacodynamic effects were comparable for the two ethnicities, with slight dose-related transient immunoglobulin (Ig)M and IgA decreases.

(B-cell-activating factor of the tumor necrosis factor (TNF) family)], and a proliferation-inducing ligand (APRIL). Both are critical factors in the maintenance of the B cell pool and humoral immunity [2]: BLyS regulates transitional stage B cell development and promotes B cell survival [3] and APRIL promotes plasmablast and plasma cell survival in the bone marrow [4-7]. Blocking these cytokines causes a reduction in mature B cells, plasma cells, and serum Igs, and also interferes with B cell development [8]. Both BLyS and APRIL have been implicated in various human autoimmune diseases, including systemic lupus erythematosus (SLE), IgA nephropathy, rheumatoid arthritis (RA), and Sjögren's syndrome [9-12].

Atacicept has shown a beneficial effect combined with an acceptable safety and tolerability profile in patients with moderate-to-severe SLE. In a post hoc analysis of the phase II/III APRIL-SLE trial, atacicept $150 \mathrm{mg}$ given once weekly subcutaneously (SC) was shown to prevent new disease flares and delay the time to first flare [13]. In the phase IIb ADDRESS II study, atacicept $150 \mathrm{mg}$ improved clinical responses versus placebo, based on SLE responder indices 4 and 6, in patients with high disease activity (defined as SLE Disease Activity Index $2000 \geq 10$ ) and in serologically active subpopulations [14].

Pharmacokinetic analyses to determine atacicept serum levels have already been carried out in clinical studies assessing single doses in healthy volunteers [15], and multiple-dose atacicept in patients with SLE, RA, multiple sclerosis, non-Hodgkin's lymphoma, multiple myeloma, and active Waldenström's macroglobulinemia [16-21]. However, pharmacokinetic data from these studies were derived using a previously validated enzyme-linked immunosorbent assay (ELISA), which was subsequently found to have inadequacies linked to the bioanalytical reference standard. Hence, a new bioanalytical ELISA for total atacicept was developed, validated, and used here and in phase 2 studies in SLE patients.

The aim of this study was to assess and compare the safety, tolerability, pharmacokinetics, and pharmacodynamics of single-dose atacicept in healthy Japanese and Caucasian subjects while generating atacicept pharmacokinetic data using the new validated bioanalytical method.

\section{Methods}

This was a phase I, randomized, double-blind, placebo-controlled, single-center, parallel-group study comparing the safety, tolerability, and the pharmacokinetic and pharmacodynamic parameters of single-dose atacicept in healthy Japanese and Caucasian subjects.

\subsection{Ethics Approval}

The protocol was approved by the Office for Research Ethics Committees Northern Ireland, and the study was conducted at the Quintiles Drug Research Unit, Guy's Hospital, in the UK (EudraCT ID: 2013-002703-34; study: EMR 700461022), in accordance with the International Conference on Harmonization Guidelines for Good Clinical Practice, the Declaration of Helsinki, the European Union Clinical Trial Directive, and all applicable local regulatory requirements. All study participants provided written informed consent.

\subsection{Study Participants}

The planned enrollment was 48 subjects: 24 Japanese and 24 Caucasian. Eligible subjects were healthy male or female Japanese or Caucasian subjects aged 18-55 years and with a body weight (BW) of 45-90 kg (female) and 55-90 kg (male) and a body mass index (BMI) of $18.0-29.9 \mathrm{~kg} / \mathrm{m}^{2}$. Japanese subjects were required to hold a valid Japanese passport, to have been born in Japan, and to have lived outside the country for $<5$ years, with both parents and all four grandparents being Japanese [22]. Caucasian subjects were required to have Caucasian parents. Caucasian subjects were matched to Japanese subjects for gender, BW $( \pm 20 \%)$, and height $( \pm 15 \%)$ at screening. Full inclusion and exclusion criteria are provided in Table S1 of the Electronic supplementary material (ESM). 


\subsection{Study Design}

Eligible Japanese subjects were randomized (1:1:1:1) to receive one single SC administration of atacicept $(25 \mathrm{mg}$, $75 \mathrm{mg}$, or $150 \mathrm{mg}$ ) or placebo using consecutive randomization codes and blinded treatment kits. Each matched Caucasian subject received the same single dose of atacicept or placebo as their Japanese counterpart. The subjects were admitted to the trial site 2 days prior to study drug administration (day 1) and discharged $48 \mathrm{~h}$ postdose. The subjects were required to return to the trial site for nine ambulatory visits between day 4 and day 42 (end of trial; Fig. S1 of the ESM).

\subsection{Objectives}

The primary objectives of this study were firstly to assess and compare the safety and tolerability of atacicept in healthy subjects of Japanese and Caucasian origin after single doses of 25,75 , or $150 \mathrm{mg}$, and secondly to compare the pharmacokinetic profiles of atacicept in the two ethnicities. Secondary objectives were to compare further pharmacokinetic parameters, markers of pharmacodynamic activity, and immunogenicity between the ethnic groups.

\subsection{Endpoints and Assessments}

\subsubsection{Safety and Tolerability}

The first primary endpoint of this study was the safety and tolerability of atacicept. Outcomes were assessed throughout the study by recording, reporting, and analyzing baseline medical conditions, treatment-emergent adverse events (TEAEs), serious adverse events (SAEs) and physical examination findings, including vital signs, standard 12-lead electrocardiogram (ECG), and laboratory tests (hematology, clinical chemistry, coagulation, urinalysis). AEs were assessed and reported according to the Qualitative Toxicity Scale and US regulations (Appendix S1 in the ESM). Local tolerability was assessed via pain scoring by each subject using a visual analogue scale ranging from 'no pain' $(0 \mathrm{~mm})$ to 'worst possible pain' $(100 \mathrm{~mm})$. Redness, bruising, swelling, and itching were assessed by the investigator or nurse using a four-point severity scale $(0=$ none, $1=$ mild, $2=$ moderate, $3=$ severe).

\subsubsection{Pharmacokinetics}

The second primary endpoint was pharmacokinetic outcomes, as measured by the area under the serum concentration-time curve from time zero to infinity $\left(\mathrm{AUC}_{0-\infty}\right)$ and the maximum observed serum concentration $\left(C_{\max }\right)$ for atacicept. If the value for the extrapolated part of $\mathrm{AUC}_{0-\infty}$
$\left(\mathrm{AUC}_{\mathrm{extra}}\right)$ provided $>20 \%$ of $\mathrm{AUC}_{0-\infty}$, the AUC from time zero to the last sampling point $\left(\mathrm{AUC}_{0-t}\right)$ was used instead. Secondary pharmacokinetic endpoints included: $\mathrm{AUC}_{0-t}$, time to reach $C_{\max }\left(t_{\max }\right)$; apparent terminal half-life $\left(t_{1 / 2}\right)$; terminal elimination rate constant $\left(\lambda_{\mathrm{z}}\right)$; apparent volume of distribution during the terminal phase $\left(V_{\mathrm{z}} / F\right)$; and apparent total clearance $(\mathrm{CL} / F)$. The pharmacokinetic parameters were evaluated as per non-compartmental analysis (NCA) standard methods, as applicable, using the computer program Pharsight Phoenix WinNonlin ${ }^{\circledR}$ version 6.3 (Certara LP, Princeton, NJ, USA).

Serum samples for pharmacokinetic analysis were obtained predose on day 1 , then postdose at $1,4,8,16$, and $24 \mathrm{~h}$ (day 2), and at $48 \mathrm{~h}$ (day 3). Additional samples were obtained at ambulatory visits on days 4, 5, 7, 10, 14, 21, 28,35 , and 42 . Pharmacokinetic samples were analyzed at the sponsor's bioanalytical laboratory, and serum atacicept levels were determined through a validated ELISA using in-house antibodies and standard Merck KGaA procedures (Appendix S2 in the ESM). In brief, samples were incubated with an acidic solution within streptavidin-coated microplate wells. A biotin-labeled antibody to detect atacicept was added concomitantly to a pH-neutralizing agent. Following a wash cycle, a horseradish peroxidase (HRP)-labeled secondary antibody against atacicept was used for detection. Quantification was performed using spectrophotometric measurements of the chromogenic substance tetramethylbenzidine (TMB), which is involved in a reaction catalyzed by HRP, resulting in a color change. The assay's lower limit of quantification (LLOQ) was $100 \mathrm{ng} / \mathrm{mL}$ of total atacicept (free and bound forms).

\subsubsection{Pharmacodynamics}

Baseline values of pharmacodynamic parameters were measured on day 1, ahead of atacicept administration (a single sampling timepoint shortly ahead of dosing was considered adequate due to negligible predose fluctuations in biomarkers). The change in pharmacodynamic parameters over prespecified intervals postdose (measured on days 2 and 42, and several timepoints in between) was a secondary endpoint. Serum $\operatorname{IgG}, \operatorname{IgA}$, and $\operatorname{IgM}$ were assessed as part of the serum chemistry panel performed by Quintiles Lab in Livingston, UK. Immune cell count, including total T cells, helper $\mathrm{T}$ cells, cytotoxic $\mathrm{T}$ cells, total $\mathrm{B}$ cells, mature naïve B cells, memory B cells, plasma cells, plasmablasts, and natural killer (NK) cells, were assessed by flow cytometry (Appendix S3 in the ESM). Free BLyS and free APRIL were only measured and assessed predose at baseline with a validated ELISA at the sponsor's bioanalytical laboratory. The assay's LLOQ was $1.56 \mathrm{ng} / \mathrm{mL}$ for BLyS and $0.3 \mathrm{ng} / \mathrm{mL}$ for APRIL. Postdose free BLyS and APRIL concentrations were not measured because no validated atacicept-compatible 
assay was available. Therefore, potential pharmacodynamic changes in these parameters were not assessable.

\subsubsection{Immunogenicity}

Atacicept immunogenicity was evaluated as an additional secondary endpoint. The incidence of subjects with antiatacicept antibodies and the antibody titer were determined predose on day 1 and on day 42 using a validated bridging ELISA, in-house antibodies, and standard Merck KGaA procedures: both atacicept-HRP and atacicept-biotin were incubated with clinical serum samples in a streptavidin-coated microtiter plate. Any anti-atacicept antibodies present in the serum formed a bridge between the atacicept-biotin already captured on the plate and the atacicept-HRP. The TMB substrate was then added and quantified by spectrophotometry. Neutralizing antibodies were only assessed if anti-atacicept antibodies were detected.

\subsection{Statistical Analysis}

Sample size was not based on power calculation. The accepted minimum number of evaluable subjects was $n=12$ [23, 24] (Japanese, $n=6$; Caucasian, $n=6$ ) per dose group, including the placebo group, resulting in a planned total of 48 subjects to be randomized to treatment. Safety and pharmacodynamic parameters were analyzed in all subjects who received the study treatment (safety analysis set). Pharmacokinetics were analyzed in subjects who received atacicept, had no major protocol deviations, and had sufficient data to determine the primary pharmacokinetic target variables (pharmacokinetics analysis set).

The primary analyses of $\mathrm{AUC}_{0-t}$ and $C_{\max }$ (log-transformed pharmacokinetic parameters) were performed using an analysis of covariance (ANCOVA) model. Ethnic group and gender were included as fixed effects, and dose (log scale) and BW were included as covariates (power model [25]). Primary pharmacokinetic parameters were log transformed for statistical analyses. The Japanese/Caucasian ratios of the geometric mean $\mathrm{AUC}_{0-t}$ and $C_{\max }$ were calculated by taking the antilogarithm of the difference between group means and corresponding $90 \%$ confidence intervals (CIs) in the log scale. The dose by ethnic group interaction was investigated using an $F$ test.

The secondary pharmacokinetic endpoints, $t_{\max }, \lambda_{\mathrm{z}}$, $t_{1 / 2}, V_{z} / F$, and CL/F, were summarized descriptively. The Hodges-Lehmann shift estimate of the Japanese-Caucasian ethnic group difference and the corresponding $90 \% \mathrm{CI}$ were computed for $t_{\max }$. Concentrations below the LLOQ were treated as zero for the computation of pharmacokinetic parameters. No adjustments for multiplicity were made.

Pharmacodynamic, safety, and tolerability outcomes were analyzed using descriptive statistics.

\subsection{Atacicept Population-Pharmacokinetics Modeling}

$\mathrm{AUC}_{\text {extra }}$ values exceeded $20 \%$ of $\mathrm{AUC}_{0-\infty}$ in all subjects (ranging from $36-47 \%$ in both ethnic groups), indicating unreliable values for all $\lambda_{\mathrm{Z}}$-derived pharmacokinetic parameters (i.e., $t_{1 / 2}, \mathrm{AUC}_{0-\infty}, \mathrm{MRT}_{0-\infty}, \mathrm{AUMC}_{0-\infty}, \mathrm{CL} / F$, and $\left.V_{\mathrm{z}} / F\right)$. As such, all $\lambda_{\mathrm{z}}$-derived pharmacokinetic parameters using NCA of observed data were excluded from the descriptive statistical analysis. Instead, $\lambda_{\mathrm{z}}$-derived pharmacokinetic parameters were calculated after "extending" the observed pharmacokinetic profiles with simulated concentrations. These simulated concentrations were obtained based on pharmacokinetic parameter estimates from individual subjects, which were derived from an atacicept-population pharmacokinetics model (a semi-mechanistic nonlinear pharmacokinetics model) that integrated data from the phase II APRIL-SLE study [13] and this study $(n=338$ [301 SLE patients and 37 healthy volunteers]; Fig. S2 and Appendix S4 of the ESM) [26]. This was a two-compartment quasi-steady-state approximation of the target-mediated drug disposition binding model with first-order absorption [27].

BW (in kg) was a significant covariate for the parameters of the population-pharmacokinetics model, CL/F, and the apparent central compartment volume of distribution $\left(V_{\mathrm{c}} / F\right)$. BW was incorporated as follows: $\mathrm{CL} / F=\mathrm{TVCL}$ $\times(\mathrm{BW} / 70)^{0.75} \times \exp \left(\eta_{\mathrm{CL}, \mathrm{i}}\right) ; V_{\mathrm{c}} / F=\mathrm{TVV}_{\mathrm{c}} \times(\mathrm{BW} / 70)^{1.00} \times$ $\exp \left(\eta_{\mathrm{Vc}, \mathrm{i}}\right)$. TVCL and $\mathrm{TVV}_{\mathrm{c}}$ are typical values of CL/F and $V_{\mathrm{c}} / F$, respectively, and $\eta_{\mathrm{CL}, \mathrm{i}}$ and $\eta_{\mathrm{Vc}, \mathrm{i}}$ are interindividual random effects, assumed to be normally distributed. The estimates of the associated exponents were close to the theoretical ones (i.e., 0.75 and 1.00, respectively), which were included in the corresponding 95\% CIs. Hence, the exponents were fixed.

\section{Results}

\subsection{Subject Disposition}

A total of 52 subjects received treatment and thus comprised the safety analysis set. Atacicept was administered to 19 Japanese subjects ( $25 \mathrm{mg}, n=7 ; 75$ and $150 \mathrm{mg}, n=6$ each); six Japanese subjects received placebo. Eighteen Caucasian subjects received atacicept ( $n=6$ in each treatment group) and nine received placebo. Subject disposition is summarized in Fig. S3 of the ESM.

One Japanese subject in the atacicept $25 \mathrm{mg}$ group was excluded from the pharmacokinetic analysis set $(n=36)$ and replaced due to a protocol deviation (tested positive for drug abuse [opiates] on treatment day 7). Three Caucasian subjects in the placebo group discontinued prematurely from the study: one due to a protocol violation and the other two 
withdrew consent for personal reasons. All withdrawals were considered to be unrelated to treatment.

\subsection{Baseline Demographics}

Baseline demographic characteristics were balanced within the specified margins for matching (Table S2 of the ESM). Mean BW was $14 \%, 9 \%$, and $14 \%$ higher in Caucasians than in Japanese subjects in the $25 \mathrm{mg}, 75 \mathrm{mg}$, and $150 \mathrm{mg}$ atacicept groups, respectively, while no difference was seen in the placebo group. Median (range) BW was 57 (45.4-78.9) $\mathrm{kg}$ in Japanese and 66 (47.2-85.6) kg in Caucasian subjects. Median (range) age was 29 (20-48) years in Japanese and 31 (18-48) years in Caucasian subjects. The two ethnic groups were similar in terms of height and BMI in both the atacicept and placebo groups, with similar numbers of males and females per group.

\subsection{Safety and Tolerability}

The number of subjects experiencing any TEAE and the frequency and type of TEAEs were comparable for the Japanese and Caucasian subjects (Table 1). Most TEAEs were mild in intensity (about $90 \%$ for both ethnicities), and the remainder were classified as moderate by the investigator. No severe TEAEs, SAEs, or deaths occurred.

Approximately half of the TEAEs reported for each ethnicity were considered to be related to treatment by the investigator. In both groups, the most frequently reported treatment-related TEAEs were upper respiratory tract infections, nasopharyngitis, and headaches. Overall, there were no clinically meaningful differences in the frequency or incidence of TEAEs, including infections, between Japanese and Caucasian subjects, and no apparent dose dependency was observed across all subjects or within ethnicities.

There were no abnormal observations during physical examinations, and no clinically significant changes from baseline in laboratory measurements, 12-lead ECG, or vital

Table 1 Summary of TEAEs by treatment group and ethnicity (safety analysis set)

\begin{tabular}{|c|c|c|c|c|c|}
\hline \multirow[t]{3}{*}{ Ethnicity } & \multirow[t]{3}{*}{ TEAE } & \multicolumn{3}{|l|}{ Atacicept } & \multirow{3}{*}{$\begin{array}{l}\text { Placebo } \\
n(\%), \mathrm{e}\end{array}$} \\
\hline & & $25 \mathrm{mg}$ & $75 \mathrm{mg}$ & $150 \mathrm{mg}$ & \\
\hline & & $n(\%), \mathrm{e}$ & $n(\%), \mathrm{e}$ & $n(\%), \mathrm{e}$ & \\
\hline \multirow[t]{12}{*}{ Japanese } & & $n=7$ & $n=6$ & $n=6$ & $n=6$ \\
\hline & All TEAEs reported in more than one subject & $4(57.1), 5$ & $2(33.3), 3$ & $4(66.7), 7$ & $1(16.7), 1$ \\
\hline & General disorders and administration site conditions & & & & \\
\hline & Fatigue & $0(0.0), 0$ & $1(16.7), 1$ & $1(16.7), 2$ & $0(0.0), 0$ \\
\hline & Infections and infestations & & & & \\
\hline & Nasopharyngitis & $0(0.0), 0$ & $1(16.7), 1$ & $1(16.7), 1$ & $0(0.0), 0$ \\
\hline & Upper respiratory tract infection & $2(28.6), 2$ & $0(0.0), 0$ & $1(16.7), 1$ & $0(0.0), 0$ \\
\hline & Nervous system disorders & & & & \\
\hline & Dizziness & $0(0.0), 0$ & $0(0.0), 0$ & $1(16.7), 1$ & $1(16.7), 1$ \\
\hline & Headache & $2(28.6), 2$ & $0(0.0), 0$ & $2(33.3), 2$ & $0(0.0), 0$ \\
\hline & Respiratory, thoracic, and mediastinal disorders & & & & \\
\hline & Oropharyngeal pain & $1(14.3), 1$ & $1(16.7), 1$ & $0(0.0), 0$ & $0(0.0), 0$ \\
\hline \multirow[t]{12}{*}{ Caucasian } & & $n=6$ & $n=6$ & $n=6$ & $n=9$ \\
\hline & All TEAEs reported in more than one subject & $1(16.7), 1$ & $2(33.3), 4$ & $3(50.0), 5$ & $4(44.4), 10$ \\
\hline & General disorders and administration site conditions & & & & \\
\hline & Fatigue & $0(0.0), 0$ & $0(0.0), 0$ & $1(16.7), 1$ & $0(0.0), 0$ \\
\hline & Infections and infestations & & & & \\
\hline & Nasopharyngitis & $1(16.7), 1$ & $2(33.3), 2$ & $0(0.0), 0$ & $3(33.3), 4$ \\
\hline & Upper respiratory tract infection & $0(0.0), 0$ & $0(0.0), 0$ & $1(16.7), 1$ & $0(0.0), 0$ \\
\hline & Nervous system disorders & & & & \\
\hline & Headache & $0(0.0), 0$ & $0(0.0), 0$ & $2(33.3), 2$ & $2(22.2), 3$ \\
\hline & Migraine & $0(0.0), 0$ & $1(16.7), 1$ & $0(0.0), 0$ & $1(11.1), 1$ \\
\hline & Gastrointestinal disorders & & & & \\
\hline & Nausea & $0(0.0), 0$ & $1(16.7), 1$ & $1(16.7), 1$ & $2(22.2), 2$ \\
\hline
\end{tabular}

$e$ events, $n$ number of subjects, TEAE treatment-emergent adverse event 
signs. Local tolerability did not differ notably between ethnicities or treatment groups. All injection-site-related pain scores were mild, and no moderate/severe pain scores were reported for any Japanese or Caucasian subjects.

\subsection{Pharmacokinetics}

Mean total atacicept serum concentration-time profiles per treatment and ethnic group (pharmacokinetic analysis set; linear scale with standard deviation) are shown in Fig. 1. Summaries of the NCA results are provided in Tables 2 and 3.

Following a single SC administration of atacicept 25, 75, or $150 \mathrm{mg}$, pharmacokinetic profiles of total atacicept in serum showed a similar multiphasic pattern in Japanese and Caucasian groups, characterized by a rapid absorption phase reaching $C_{\max }$ after about $20-60 \mathrm{~h}$ (median $t_{\max }$ ), a distribution phase lasting 7-10 days, and a long terminal elimination phase (Fig. 1a).

The non-compartmental pharmacokinetic evaluation of all individual concentration-time profiles resulted in reliable estimation of the pharmacokinetic parameters of atacicept: $C_{\text {max }}, t_{\text {max }}$, and $\mathrm{AUC}_{0-t}($ Table 2$)$.

\subsubsection{Primary Pharmacokinetic Endpoints: $A U C_{0-\mathrm{t}}$ and $C_{\max }$}

In the Japanese and Caucasian groups, atacicept $C_{\max }$ increased in a less than dose-proportional manner across the tested dose range. Most notably, however, in Japanese subjects, the geometric mean $C_{\max }$ was approximately $10 \%$ (at $25 \mathrm{mg}$ ), 18\% (at $75 \mathrm{mg}$ ), and 55\% (at $150 \mathrm{mg}$ ) higher than in Caucasian subjects (Fig. 1a). The marked increase in $C_{\max }$ in the Japanese $150 \mathrm{mg}$ group was due to a single subject included in the pharmacokinetic analysis set who was considered an outlier. After excluding this subject from the analysis, the increase in $C_{\max }$ in Japanese subjects in the $150 \mathrm{mg}$ dose group was reduced to $15 \%$ versus Caucasian subjects (Fig. 1b).

Overall exposure, based on the geometric mean $\mathrm{AUC}_{0-t}$ and the geometric mean $C_{\max }$, was slightly higher in Japanese subjects than in Caucasian subjects (Table 2), likely due to differences in BW, albeit within the prespecified margin of $\pm 20 \%$ (Table S2 of the ESM). When the differences in BW were taken into account using an ANCOVA model, weight-adjusted atacicept exposure in Japanese and Caucasian subjects was comparable $\left(\mathrm{AUC}_{0-t}\right.$ ratio of $107.21 \%$ [90\% CI 93.42-123.02\%] and $C_{\max }$ ratio of $95.74 \%$ [90\% CI 74.26-123.43\%]) across all dose levels (Table 3).

The dose-pharmacokinetics relationship, based on AUC ${ }_{0-t}$ and $C_{\text {max }}$, showed a decrease in dose-normalized $\mathrm{AUC}_{0-t}$ $\left(\mathrm{AUC}_{0-l} / \mathrm{dose}\right)$ and a slight decrease in dose-normalized $C_{\max }$ $\left(C_{\max } /\right.$ dose $)$ with increasing dose, and was comparable for Japanese and Caucasian subjects (Table 4). An ANCOVA showed nonproportional pharmacokinetics in Caucasian and Japanese subjects, and the results were confirmed by a power model (Table S3 of the ESM). The $F$ test for the dose by ethnic group interaction resulted in nonsignificant $p$ values for $\mathrm{AUC}_{0-t}(p=0.24)$ and $C_{\text {max }}(p=0.29)$, thus not supporting an assumption of differences between Japanese and Caucasian subjects in the dose-pharmacokinetics relationship.

\subsubsection{Secondary Pharmacokinetic Endpoints: $t_{\text {max }}, \lambda_{z^{\prime}} t_{1 / 2}$, $V_{\mathrm{z}} / F$, and $\mathrm{CL} / F$}

Median $t_{\max }$ values ranged from 20 to $48 \mathrm{~h}$ in Japanese subjects and from 36 to $60 \mathrm{~h}$ in Caucasian subjects, respectively (Table 2). The median difference in $t_{\max }$ between Japanese and Caucasian subjects was $\sim 8 \mathrm{~h}(p=0.3985)$, which was not considered clinically relevant.

Based on the simulated pharmacokinetic data $\left(\lambda_{\mathrm{z}}\right.$-derived outcomes; Table 5), no significant differences in pharmacokinetics were detected between Japanese and Caucasian subjects. Once BW differences were accounted for, the effect of ethnicity on CL/F and $V_{\mathrm{z}} / F$ was insignificant: it was associated with decreases of only 1 and 1.2 units in the objective function $(p>0.27)$.

The mean terminal $t_{1 / 2}$ was simulated to be 36,35 , and 35 days in the 25,75 , and $150 \mathrm{mg}$ treatment groups for Caucasian subjects, respectively, and 36, 34, and 36 days in the 25, 75, and $150 \mathrm{mg}$ treatment groups for Japanese subjects, respectively (Table 5).

\subsection{Pharmacodynamics}

In both the Japanese and Caucasian subjects, transient decreases from baseline in serum mean IgA were observed: 4-7\% decreases at 7 days after atacicept $25 \mathrm{mg}$, and $8-12 \%$ decreases at 14-28 days after atacicept 75 or $150 \mathrm{mg}$ (Fig. 2a). Mean serum IgM levels decreased from baseline by about $14-18 \%$ as early as 14 days after single-dose administration of 25, 75, or $150 \mathrm{mg}$ atacicept (Fig. 2b). Decreases in serum mean IgA and mean IgM were transient but the duration appeared to prolong with increasing dose. Mean serum IgA levels were fully recovered at approximately day 28 post atacicept $25 \mathrm{mg}$, and partially recovered at day 42 following a single dose of atacicept 75 or $150 \mathrm{mg}$, while mean serum IgM levels remained reduced at day 42 after atacicept $150 \mathrm{mg}$ (Fig. 2; Fig. S4 of the ESM). There was no apparent treatment effect on serum IgG levels (not shown). After the placebo treatment, no notable trends in any of the Ig levels were observed.

Atacicept was associated with no notable alterations in blood immune cell count for $\mathrm{T}$ cells $\left(\mathrm{CD} 3^{+} \mathrm{CD} 4^{+}\right.$ or $\left.\mathrm{CD}^{+} \mathrm{CD}^{+}\right)$or total $\mathrm{NK}$ cells $\left(\mathrm{CD} 56^{+} \mathrm{CD} 16^{+}\right)$. There were slight, transient increases in total $\mathrm{B}$ cells $\left(\mathrm{CD} 19^{+} \mathrm{CD} 45^{+} \mathrm{CD}^{-}\right.$) (Fig. 3), mature naïve B cells 
Fig. 1 Mean serum concentration-time profiles of total atacicept by atacicept dose and ethnicity: a pharmacokinetic analysis set; $\mathbf{b}$ pharmacokinetic analysis set without outlier subject
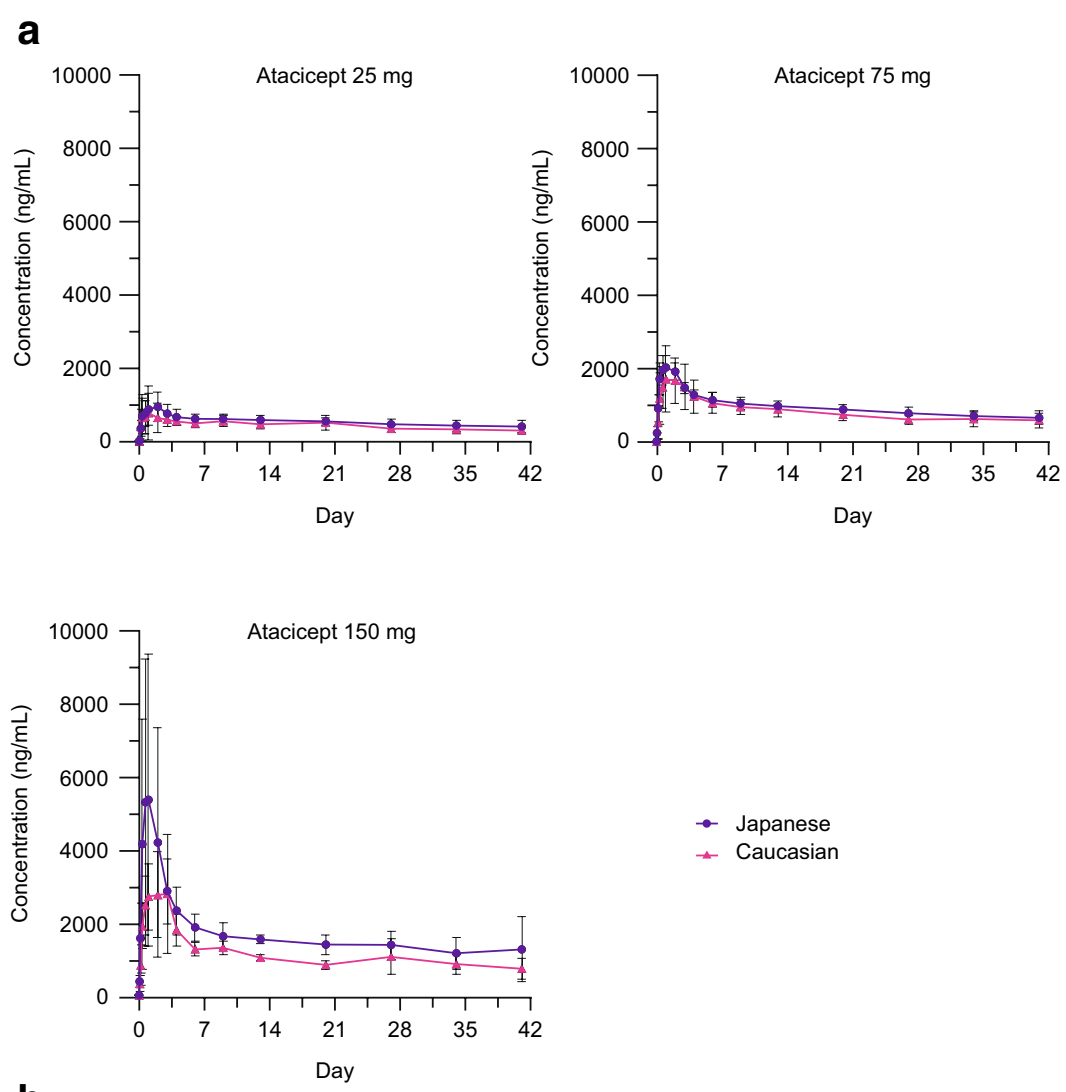

b
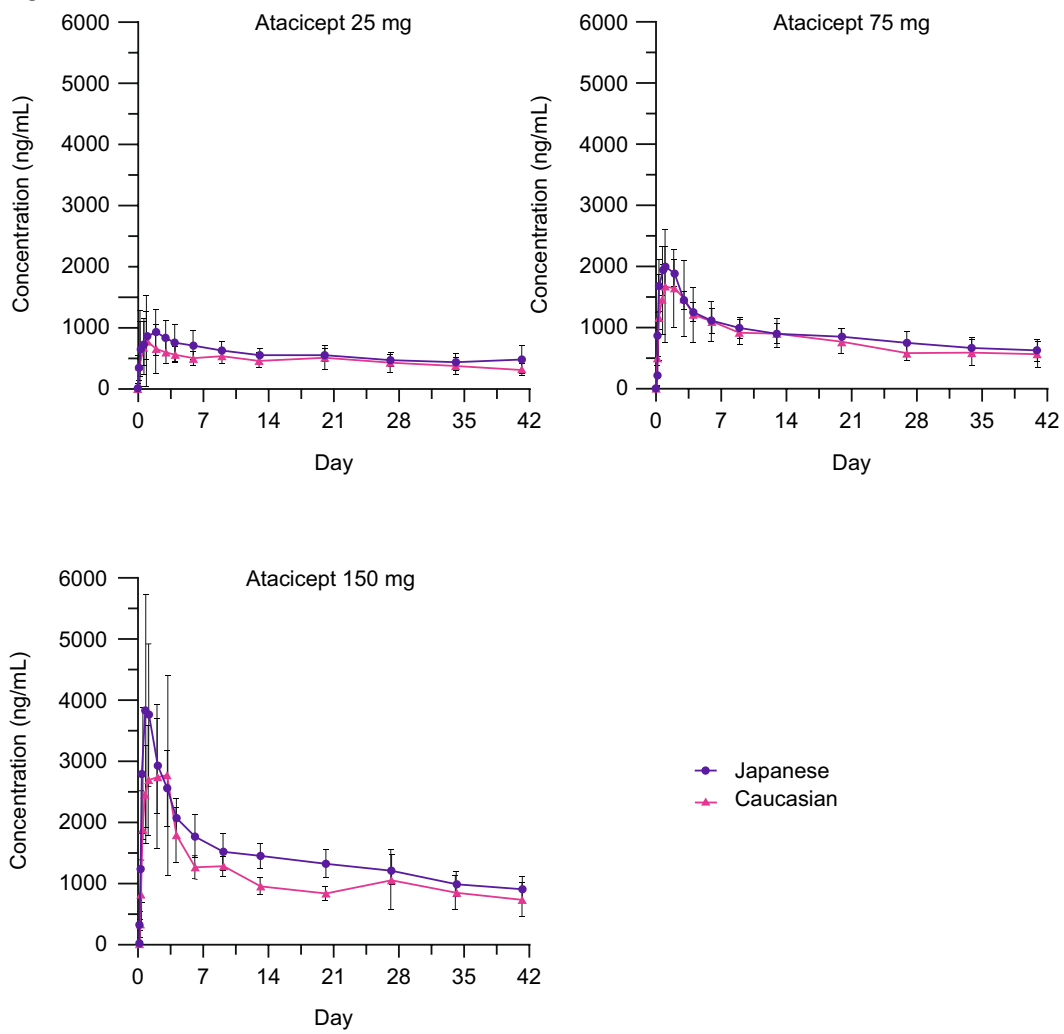

$\rightarrow$ Japanese

$\star$ Caucasian

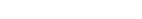

Day 
Table 2 Atacicept pharmacokinetic parameters (geometric mean $C_{\max }$, geometric mean $\mathrm{AUC}_{0-t}$, median $t_{\max }$ ) by atacicept dose and ethnicity derived from NCA (pharmacokinetic analysis set)

\begin{tabular}{|c|c|c|c|c|c|c|c|c|c|}
\hline \multirow{2}{*}{$\begin{array}{l}\text { Pharma- } \\
\text { cokinetic } \\
\text { parameter }^{\mathrm{a}}\end{array}$} & \multicolumn{3}{|l|}{$25 \mathrm{mg}$} & \multicolumn{3}{|l|}{$75 \mathrm{mg}$} & \multicolumn{3}{|l|}{$150 \mathrm{mg}$} \\
\hline & $\begin{array}{l}\text { Japanese } \\
n=6\end{array}$ & $\begin{array}{l}\text { Caucasian } \\
n=6\end{array}$ & Ratio $^{\mathrm{b}}$ & $\begin{array}{l}\text { Japanese } \\
n=6\end{array}$ & $\begin{array}{l}\text { Caucasian } \\
n=6\end{array}$ & Ratio $^{\mathrm{b}}$ & $\begin{array}{l}\text { Japanese } \\
n=6\end{array}$ & $\begin{array}{l}\text { Caucasian } \\
n=6\end{array}$ & Ratio $^{b}$ \\
\hline \multicolumn{10}{|c|}{$C_{\max }(\mathrm{ng} / \mathrm{mL})$} \\
\hline Geo mean & 921 & 835 & 1.10 & 2079 & 1762 & 1.18 & 4843 & 3133 & 1.55 \\
\hline Geo CV\% & 48.9 & 56.3 & & 18.4 & 49.5 & & 63 & 50.4 & \\
\hline $95 \% \mathrm{CI}$ & $567-1498$ & $481-1477$ & & $1717-2518$ & $1078-2880$ & & $2640-8884$ & $1902-5161$ & \\
\hline \multicolumn{10}{|c|}{$\mathrm{AUC}_{0-t}($ day $\cdot \mathrm{ng} / \mathrm{mL})$} \\
\hline Geo mean & 21,343 & 18,181 & 1.17 & 37,679 & 33,930 & 1.11 & 65,221 & 44,689 & 1.46 \\
\hline Geo CV\% & 23.8 & 27.2 & & 14.4 & 28 & & 33 & 26.5 & \\
\hline $95 \% \mathrm{CI}$ & $16,677-27,314$ & $13,735-24,065$ & & $32,417-43,796$ & $25,433-45,266$ & & $46,530-91,421$ & $33,994-58,748$ & \\
\hline \multicolumn{10}{|l|}{$t_{\max }$ (hours) } \\
\hline Median & 48 & 36 & & 36 & 60 & & 20 & 36 & \\
\hline Range & $8-144$ & $16-480$ & & $16-48$ & $16-144$ & & $16-24$ & $16-72$ & \\
\hline
\end{tabular}

$A U C_{0-\infty}$ area under the serum concentration-time curve from time zero to infinity, $A U C_{0-t}$ area under the serum concentration-time curve from time zero to the last sampling point, $A U C_{\text {extra }}$ extrapolated part of $\mathrm{AUC}_{0-\infty}, C I$ confidence interval, $C L / F$ apparent total clearance, $C_{m a x}$ maximum observed serum concentration, GeoCV\% geometric mean coefficient of variation, Geo mean geometric mean, NCA non-compartmental analysis, $t_{1 / 2}$ apparent terminal half-life, $t_{\max }$ time to reach maximum observed serum concentration, $V_{z} / F$ apparent volume of distribution during the terminal phase, $\lambda_{z}$ terminal elimination rate constant

${ }^{\mathrm{a}}$ All subjects presented $\mathrm{AUC}_{\text {extra }}$ values higher than $20 \%$ of $\mathrm{AUC}_{0-\infty}$, so all values derived using $\lambda_{\mathrm{z}}\left(t_{1 / 2}, \mathrm{CL} / F\right.$ and $\left.V_{\mathrm{z}} / F\right)$ and $\mathrm{AUC} C_{0-\infty}$ have to be regarded as not fully reliable and were excluded from the descriptive statistical analysis

bJapanese:Caucasian

Table 3 Comparison of the geometric least square means of $C_{\max }$ and $\mathrm{AUC}_{0-t}$ between ethnicities, adjusted for dose and body weight ${ }^{\mathrm{a}}$ (pharmacokinetic analysis set)

\begin{tabular}{lll}
\hline Pharmacokinetic parameter & $\begin{array}{l}\text { Japanese } \\
n=18\end{array}$ & $\begin{array}{l}\text { Caucasian } \\
n=18\end{array}$ \\
\hline $\mathrm{AUC}_{0-t}$, day·ng/mL & & \\
Geometric LSM across all dose levels (95\% CI) & $34,820(31,210-38,840)$ & $32,480(29,120-36,230)$ \\
Japanese/Caucasian ratio, \% (90\% CI) & $107.21(93.42-123.02)$ & \\
$C_{\text {max }}$, ng/mL & & \\
Geometric LSM across all dose levels (95\% CI) & $1829(1495-2238)$ & $1911(1562-2338)$ \\
Japanese/Caucasian ratio, \% (90\% CI) & $95.74(74.26-123.43)$ & \\
\hline
\end{tabular}

${ }^{a}$ Results based on an ANCOVA model for log-transformed pharmacokinetic parameters with ethnic group and gender as fixed effects plus $\log ($ dose $)$ and body weight as covariates

$A N C O V A$ analysis of covariance, $A U C_{0-t}$ area under the serum concentration-time curve from time zero to the last sampling point, $C I$ confidence interval, $C_{\max }$ maximum observed serum concentration, $L S M$ least square mean
$\left(\mathrm{CD} 19+\mathrm{CD} 20+\operatorname{IgD}+\mathrm{CD} 27^{-}\right)$, and memory B cells in both ethnicities and in all atacicept and placebo groups (Fig. S5 of the ESM). Increases usually occurred within the first 4 days postdose, followed by a decline to predose levels, without an apparent dose dependency. The initial increase in cell number appeared to be more visible in the groups that received atacicept.

Median baseline free BLyS and APRIL concentrations were similar and at the low end of the respective assay LLOQ across all treatment groups and for both ethnicities. Postdose free BLyS and APRIL concentrations were not measured.

\subsection{Immunogenicity}

No anti-atacicept antibodies were detected in any subject.

\section{Discussion}

This study in healthy volunteers aimed to compare the safety, tolerability, pharmacokinetics, and pharmacodynamics of single-dose SC atacicept in Japanese and Caucasian subjects. 
Table 4 Median dosenormalized $C_{\max }$ and $\mathrm{AUC}_{0-t}$ by atacicept dose and ethnicity (pharmacokinetic analysis set)

\begin{tabular}{|c|c|c|c|c|c|c|}
\hline \multirow{2}{*}{$\begin{array}{l}\text { Pharma- } \\
\text { cokinetic } \\
\text { parameter }\end{array}$} & \multicolumn{2}{|l|}{$25 \mathrm{mg}$} & \multicolumn{2}{|l|}{$75 \mathrm{mg}$} & \multicolumn{2}{|l|}{$150 \mathrm{mg}$} \\
\hline & $\begin{array}{l}\text { Japanese } \\
n=7\end{array}$ & $\begin{array}{l}\text { Caucasian } \\
n=6\end{array}$ & $\begin{array}{l}\text { Japanese } \\
n=6\end{array}$ & $\begin{array}{l}\text { Caucasian } \\
n=6\end{array}$ & $\begin{array}{l}\text { Japanese } \\
n=6\end{array}$ & $\begin{array}{l}\text { Caucasian } \\
n=6\end{array}$ \\
\hline \multicolumn{7}{|c|}{$C_{\max } /$ dose, $\mathrm{ng} / \mathrm{mL} / \mathrm{mg}$} \\
\hline Median $^{\mathrm{a}}$ & 35.4 & 29.5 & 28.2 & 23.5 & 27.1 & 20.1 \\
\hline Range & $21.4-62.0$ & $19.9-90.8$ & $20.4-35.1$ & $11.2-46.0$ & $19.3-87.8$ & $11.0-39.2$ \\
\hline \multicolumn{7}{|c|}{$\mathrm{AUC}_{0-t} / \mathrm{dose}$, day $\mathrm{ng} / \mathrm{mL} / \mathrm{mg}$} \\
\hline Median $^{\mathrm{a}}$ & 871.1 & 740.6 & 495.3 & 466.9 & 418.4 & 312.6 \\
\hline Range & $590.3-1155.1$ & $482.6-1081.3$ & $424.6-594.1$ & $306.6-693.4$ & $319.5-775.0$ & 196.6-406.9 \\
\hline
\end{tabular}

$A U C_{0-t}$ area under the serum concentration-time curve from time zero to the last sampling point, $C_{\max }$ maximum observed serum concentration

${ }^{\text {a } D o s e ~ n o r m a l i z e d ~}$
There were no safety concerns or unexpected tolerability findings, including local tolerability parameters, across the tested dose levels of atacicept and ethnicities during this study. Moreover, vital signs, ECG, and laboratory parameters did not show any clinically relevant trends or changes. TEAEs were mainly of mild (90\%) and moderate intensity, were reversible, and resolved without any clinical sequelae. Comparable to an earlier single-dose escalation study in healthy volunteers [15], there was no apparent relationship between atacicept dose and TEAE incidence. Overall, there were no clinically meaningful differences in atacicept safety and tolerability profiles between Japanese and Caucasian subjects.

Atacicept exposure, based on the geometric mean $C_{\max }$ and $\mathrm{AUC}_{0-t}$, was slightly higher in Japanese subjects than in Caucasian subjects. Notably, there was a marked increase (by 55\%) in $C_{\max }$ in the Japanese $150 \mathrm{mg}$ group. This was due to a single subject who was considered an outlier and was not associated with any untoward TEAEs. After excluding this subject from the analysis, the increase in $C_{\max }$ in Japanese subjects in the $150 \mathrm{mg}$ dose group was reduced to $15 \%$ and was thereby comparable to the $C_{\max }$ increases (10-18\%) observed at the two lower atacicept doses.

Generally, Caucasian subjects had higher BWs than their Japanese counterparts; the differences in BW lay within the study's prespecified margin of $\pm 20 \%$ and reflected known national differences of approximately $18 \%$ between subjects from Japan and the UK [28, 29]. After adjusting for BW, atacicept exposure was shown to be comparable between ethnicities and across doses. A decrease in dose-normalized $\mathrm{AUC}_{0-t}$ with increasing atacicept dose and a slight decrease in dose-normalized $C_{\max }$ indicated nonproportional pharmacokinetics over the dose range in both ethnicities.

Consistent with the inhibitory activity of atacicept on BLyS and APRIL, decreases from baseline in mean serum IgA and IgM were observed across atacicept doses and ethnicities. Decreases were more sustained with increasing dose but were transient. By contrast, there was no apparent reduction in serum IgG levels following single-dose atacicept at any of the tested doses. These observations were consistent with data from an earlier single-dose escalation study of up to $630 \mathrm{mg}$ atacicept in healthy volunteers [15]. An explanation for the lack of decrease in IgG levels following single-dose atacicept could be the differences in intrinsic half-lives and relative abundances of $\operatorname{IgG}, \operatorname{IgA}$, and $\operatorname{IgM}$ in serum: $\operatorname{IgG}$, with its 4 subtypes $\left(\operatorname{IgG}_{1-4}\right)$, accounts for about $75-80 \%$ of the serum antibodies in humans and has an overall half-life of about 21 days (except for $\mathrm{IgG}_{3}$ ) [30], while $\operatorname{IgM}$ and $\operatorname{IgA}$ have much shorter half-lives of about 5 and 6 days, respectively. Accordingly, previous multiple-dose studies with atacicept demonstrated pronounced reductions not only in $\operatorname{IgA}$ and $\operatorname{IgM}$, but also in IgG, in SLE and RA patients [13, 18, 20, 31, 32].

There were no clinically relevant changes in immune cell counts over time following single-dose atacicept administration in any treatment group. There were some minor transient increases in total $\mathrm{CD}^{+} \mathrm{CD}^{+}$and $\mathrm{CD} 3^{+} \mathrm{CD} 8^{+} \mathrm{T}$ cells and total $\mathrm{CD} 56^{+} \mathrm{CD} 16^{+} \mathrm{NK}$ cells that were likely due to normal variation. Immune-cell counts for plasma cells and plasmablasts were too low for evaluation, but there were slight increases in total and subsets of B cells (both mature naïve $B$ cells and memory $B$ cells) that were associated with single-dose atacicept administration in both ethnicities, with no apparent dose dependency. These early increases (usually within the first 4 days postdose) were followed by a decline to predose levels. This was consistent with previous observations of initial $\mathrm{B}$ cell increases in $\mathrm{CD} 27^{+}$class-switched memory B cells with both atacicept and belimumab [16, 17, 33-36]. Antibodies to atacicept were tested at predose and at day 42 following dosing (end of treatment) as part of the safety assessments. No anti-atacicept antibodies were detected in this single-dose study. Whilst this study was not intended to and cannot predict potential long-term immunogenicity with atacicept following multiple dosing, a low immunogenicity is expected with atacicept due to its mode of action and the effect of reducing $B$ cells and plasma cells. 
Table 5 Pharmacokinetic parameters by atacicept dose and ethnicity, derived from a semi-mechanistic nonlinear population-pharmacokinetic model

\begin{tabular}{|c|c|c|c|c|c|c|}
\hline \multirow{3}{*}{$\begin{array}{l}\text { Pharmacoki- } \\
\text { netic param- } \\
\text { eters }\end{array}$} & \multicolumn{2}{|l|}{$25 \mathrm{mg}$} & \multicolumn{2}{|l|}{$75 \mathrm{mg}$} & \multicolumn{2}{|l|}{$150 \mathrm{mg}$} \\
\hline & Japanese & Caucasian & Japanese & Caucasian & Japanese & Caucasian \\
\hline & $n=7^{\mathrm{a}}$ & $n=6$ & $n=6$ & $n=6$ & $n=6$ & $n=6$ \\
\hline \multicolumn{7}{|l|}{$\lambda_{\mathrm{z}}\left(\right.$ day $\left.^{-1}\right)$} \\
\hline Mean & 0.020 & 0.019 & 0.021 & 0.020 & 0.019 & 0.020 \\
\hline Median & 0.019 & 0.019 & 0.020 & 0.020 & 0.020 & 0.021 \\
\hline Range & $0.016-0.031$ & $0.017-0.022$ & $0.018-0.025$ & $0.018-0.024$ & $0.016-0.022$ & $0.016-0.023$ \\
\hline \multicolumn{7}{|l|}{$t_{1 / 2}$ (days) $)^{\mathrm{b}}$} \\
\hline Mean & 36 & 36 & 34 & 35 & 36 & 35 \\
\hline Median & 36 & 36 & 35 & 35 & 35 & 34 \\
\hline Range & $22-43$ & $31-41$ & $28-38$ & $29-39$ & $31-45$ & $30-43$ \\
\hline \multicolumn{7}{|l|}{$V_{\mathrm{z}} / F(\mathrm{~L})$} \\
\hline Mean & 36 & 46 & 62 & 73 & 76 & 106 \\
\hline Median & 31 & 45 & 63 & 69 & 79 & 99 \\
\hline Range & $26-57$ & $25-68$ & $53-72$ & $42-111$ & $34-100$ & 69-166 \\
\hline \multicolumn{7}{|l|}{ CL/F (L/hour) } \\
\hline Mean & 0.0301 & 0.0374 & 0.0540 & 0.0611 & 0.0624 & 0.0911 \\
\hline Median & 0.0254 & 0.0320 & 0.0564 & 0.0568 & 0.0650 & 0.0863 \\
\hline Range & $0.0213-0.0442$ & $0.0215-0.0632$ & $0.0407-0.0697$ & $0.0352-0.0845$ & $0.0223-0.0915$ & $0.0470-0.1471$ \\
\hline \multicolumn{7}{|c|}{$\mathrm{AUC}_{0-\alpha}($ day $\cdot \mathrm{ng} / \mathrm{mL})$} \\
\hline Mean & 37,115 & 31,111 & 60,175 & 55,527 & 122,650 & 79,151 \\
\hline Median & 40,945 & 32,557 & 55,387 & 55,019 & 96,854 & 75,314 \\
\hline Range & $23,582-48,832$ & $16,479-48,449$ & $44,853-76,824$ & $36,992-88,903$ & $68,294-280,143$ & $42,497-132,879$ \\
\hline \multicolumn{7}{|l|}{$\mathrm{MRT}_{0-\alpha}$ (day) } \\
\hline Mean & 47 & 47 & 44 & 44 & 48 & 46 \\
\hline Median & 47 & 49 & 45 & 46 & 42 & 44 \\
\hline Range & $27-59$ & $35-55$ & $35-51$ & $36-50$ & $37-69$ & $36-64$ \\
\hline \multicolumn{7}{|c|}{$\mathrm{AUMC}_{0-\alpha}\left(\right.$ day $\left.^{2} \cdot \mathrm{ng} / \mathrm{mL}\right)$} \\
\hline Mean & $1,780,324$ & $1,519,422$ & $2,691,422$ & $2,481,129$ & $6,535,588$ & $3,965,695$ \\
\hline Median & $1,926,887$ & $1,661,765$ & $2,405,778$ & $2,286,035$ & $4,486,640$ & $3,387,582$ \\
\hline Range & $708,360-2,545,213$ & $580,936-2,431,350$ & $\begin{array}{r}1,591,190- \\
3,935,417\end{array}$ & $\begin{array}{r}1,391,732- \\
4,282,241\end{array}$ & $\begin{array}{r}2,558,776- \\
19,458,885\end{array}$ & $\begin{array}{r}1,621,036- \\
8,566,427\end{array}$ \\
\hline
\end{tabular}

$\lambda_{z}$ terminal rate constant, $A U C_{0-\infty}$ area under the serum concentration-time curve from time zero to infinity, $C L / F$ apparent total clearance, $t_{1 / 2}$ apparent terminal half-life, $V_{z} / F$ apparent volume of distribution during the terminal phase

${ }^{a}$ One more subject than in the pharmacokinetic analysis set $(n=36)$ was included for the atacicept population-pharmacokinetic model. This subject was excluded from the pharmacokinetic analysis set due to a positive test for opiate use, which was defined as an exclusion criterion in this study, on treatment day 7. However, the subject was included for the model because the pharmacokinetic data collected were still considered valid and unlikely to be affected by the opiate use

${ }^{\mathrm{b}} t_{1 / 2}$ values (in days) based on the invalidated bioanalytical assay published by Munafo et al. in 2007 [15]: atacicept $2.1 \mathrm{mg}(n=5)$, mean: 8.0, median: 7.4 (range: $1.9-15.3)$; atacicept $70 \mathrm{mg}(n=5), 10.6,11.0(9.10-11.5)$; atacicept $210 \mathrm{mg}(n=5), 15.9,18.0(7.00-19.7)$; atacicept 630 mg $(n=4), 12.8,13.0(12.0-13.4)$

In the 52-week APRIL-SLE study, anti-atacicept antibodies were detected in 3 of 301 (1.0\%) atacicept-treated SLE patients at the 24-week follow-up visit [13]. In the 24-week ADDRESS II study, 8 of 206 (3.9\%) atacicept-treated SLE patients had detectable anti-atacicept antibodies [14].

This study had several limitations. Firstly, this was a single-dose study in healthy subjects. As such, the observed safety and pharmacodynamic outcomes with atacicept may not reflect the effects of multiple dosing in patients with autoimmune disease. Secondly, $\lambda_{z}$-derived pharmacokinetic parameters could not be reliably calculated by NCA for atacicept, as $\mathrm{AUC}_{\text {extra }}$ was $>20 \%$ of $\mathrm{AUC}_{0-\infty}$. Of note, when designing this study, the implemented pharmacokinetic sampling time points up to 42 days postdose (end-of-trial visit) were deemed to be adequate considering data from the earlier single-dose escalation study in healthy volunteers [15], 

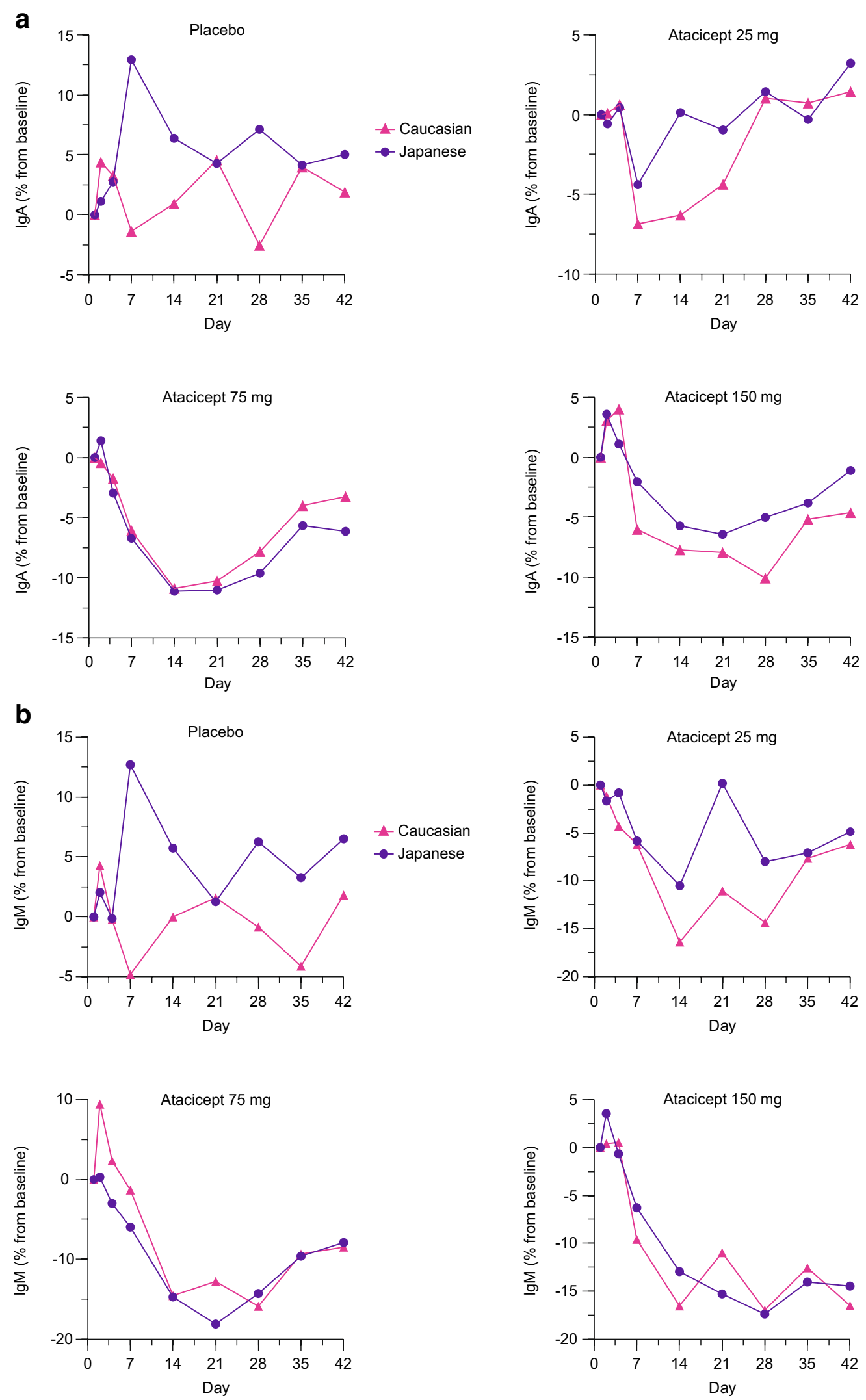

Fig. 2 a Change in mean IgA concentration from baseline to day 42 by atacicept dose and ethnicity (linear scale). b Change in mean IgM concentration from baseline to day 42 by atacicept dose and ethnicity (linear scale) 

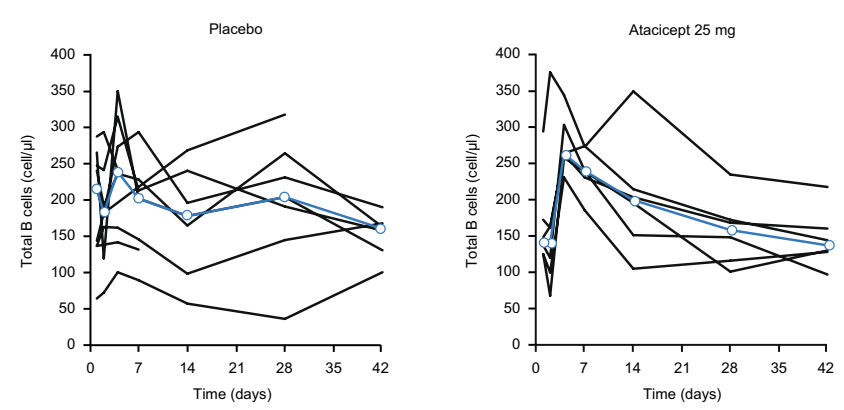

Caucasian
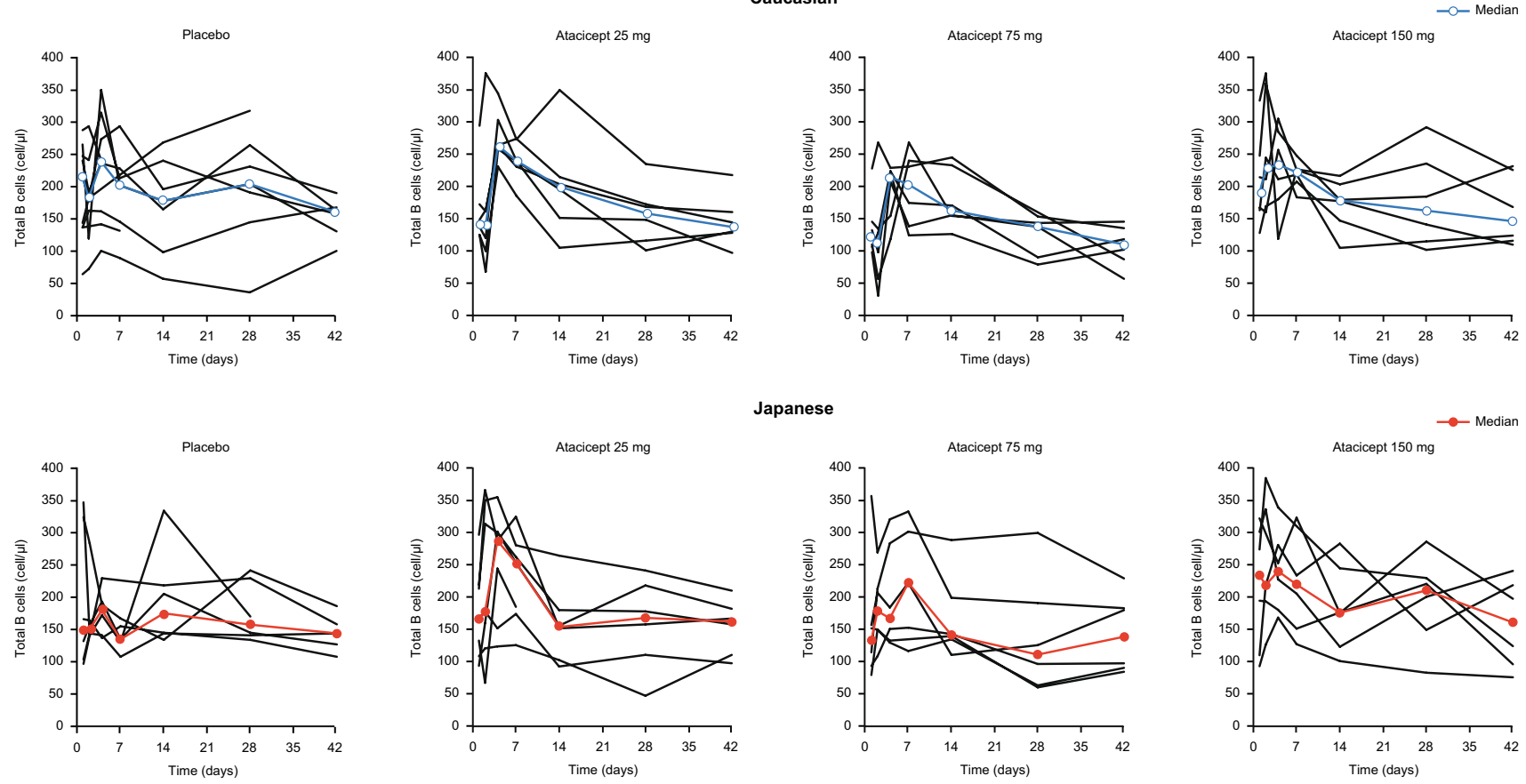

Fig. 3 Individual and median total B cell $\left(\mathrm{CD} 19^{+} \mathrm{CD} 45^{+} \mathrm{CD} 3^{-}\right)$counts over time by treatment group and ethnicity (safety analysis set). Median total B cell $\left(\mathrm{CD} 19^{+} \mathrm{CD}_{4} 5^{+} \mathrm{CD}^{-}\right)$counts for the treatment groups are indicated by the thicker (red and blue) curves

in which free atacicept was found to have a median $t_{1 / 2}$ of 12.4 days. Further, it is currently unknown how endogenous APRIL and BLyS bound to atacicept might affect $t_{1 / 2}$. In this study, only 'total' atacicept could be measured using a dissociation assay. Experiments and modeling to predict $t_{1 / 2}$ of bound versus unbound atacicept are ongoing and will be discussed in future publications.

\section{Conclusion}

In conclusion, single-dose atacicept presented an acceptable safety and tolerability profile with no differences between Japanese and Caucasian subjects, and no anti-atacicept antibodies were detected. Pharmacokinetic parameters were shown to be comparable for the two ethnicities after adjustment for BW. Changes in pharmacodynamic markers were also comparable.

Acknowledgements The authors confirm that the principal investigator for this paper was Tim Mant, BSc, FRCP, FFPM, Quintiles Drug Research Unit at Guy's Hospital, London, UK, and that he had direct responsibility for the conduct of the clinical trial in accordance with the clinical trial protocol and protocol amendment, and in compliance with Good Clinical Practice and all applicable regulatory requirements. The authors would like to thank the volunteers and their families, investigators, co-investigators, and the study teams at each of the participating centers. In addition, the authors thank Melanie Jones, Muchaala Yeboah, and Hannah Fleetwood of Bioscript Science, Macclesfield, UK for providing medical writing support, which was funded by Merck
KGaA, Darmstadt, Germany, in accordance with Good Publication Practice (GPP3) guidelines (http://www.ismpp.org/gpp3).

Author contributions DW, WU, PW, and ÖY were involved in the conception and design of the study and all authors were involved in the preparation of the manuscript. OP performed the pharmacokinetic modeling and simulation analyses. The final version was approved by all authors.

\section{Compliance with Ethical Standards}

Funding This study was sponsored by Merck KGaA, Darmstadt, Germany.

Conflicts of interest Daniela Willen, Wolfgang Uhl and Peter Wolna were employees of Merck KGaA, Darmstadt, Germany at the time of this study. Özkan Yalkinoglu is an employee of Merck KGaA, Darmstadt, Germany. Orestis Papasouliotis is an employee of Merck Institute for Pharmacometrics, Lausanne, Switzerland, an affiliate of Merck KGaA, Darmstadt, Germany.

Ethical approval The protocol was approved by the Office for Research Ethics Committees Northern Ireland and the study was conducted at the Quintiles Drug Research Unit, Guy's Hospital, in the UK (EudraCT ID: 2013-002703-34; study: EMR 700461-022), in accordance with the International Conference on Harmonization Guidelines for Good Clinical Practice, the Declaration of Helsinki, the European Union Clinical Trial Directive, and all applicable local regulatory requirements.

Data sharing statement Atacicept is under clinical investigation and has not yet been approved in any sought-after indication by any health authority worldwide. Therefore, there is no plan for data sharing at this point in time. Please note that for all new products or new indica- 
tions approved in both the European Union and the United States after January 1, 2014, Merck will share patient level, study level data after de-identification, as well as redacted study protocols and clinical study reports from clinical trials in patients. These data will be shared with qualified scientific and medical researchers, upon researcher's request, as necessary for conducting legitimate research. Such requests must be submitted in writing to the company's data sharing portal and will be internally reviewed regarding criteria for researcher qualifications and legitimacy of the research purpose (https://www.merckgroup.com/en/ research/our-approach-to-research-and-development/healthcare/clini cal-trials/commitment-responsible-data-sharing.html).

Open Access This article is distributed under the terms of the Creative Commons Attribution-NonCommercial 4.0 International License (http://creativecommons.org/licenses/by-nc/4.0/), which permits any noncommercial use, distribution, and reproduction in any medium, provided you give appropriate credit to the original author(s) and the source, provide a link to the Creative Commons license, and indicate if changes were made.

\section{References}

1. Samy E, Wax S, Huard B, Hess H, Schneider P. Targeting BAFF and APRIL in systemic lupus erythematosus and other antibodyassociated diseases. Int Rev Immunol. 2017;36(1):3-19.

2. Dillon SR, Harder B, Lewis KB, Moore MD, Liu H, Bukowski $\mathrm{TR}$, et al. B-lymphocyte stimulator/a proliferation-inducing ligand heterotrimers are elevated in the sera of patients with autoimmune disease and are neutralized by atacicept and B-cell maturation antigen-immunoglobulin. Arthritis Res Ther. 2010;12(2):R48.

3. Schiemann B, Gommerman JL, Vora K, Cachero TG, ShulgaMorskaya S, Dobles M, et al. An essential role for BAFF in the normal development of B cells through a BCMA-independent pathway. Science. 2001;293(5537):2111-4.

4. Belnoue E, Pihlgren M, McGaha TL, Tougne C, Rochat AF, Bossen C, et al. APRIL is critical for plasmablast survival in the bone marrow and poorly expressed by early-life bone marrow stromal cells. Blood. 2008;111(5):2755-64.

5. Matthes T, Dunand-Sauthier I, Santiago-Raber ML, Krause $\mathrm{KH}$, Donze O, Passweg J, et al. Production of the plasma-cell survival factor a proliferation-inducing ligand (APRIL) peaks in myeloid precursor cells from human bone marrow. Blood. 2011;118(7):1838-44.

6. Haselmayer P, Vigolo M, Nys J, Schneider P, Hess H. A mouse model of systemic lupus erythematosus responds better to soluble TACI than to soluble BAFFR, correlating with depletion of plasma cells. Eur J Immunol. 2017;47(6):1075-85.

7. Tran NL, Schneider P, Santiago-Raber ML. TACI-dependent APRIL signaling maintains autoreactive B cells in a mouse model of systemic lupus erythematosus. Eur J Immunol. 2017;47(4):713-23.

8. Gross JA, Dillon SR, Mudri S, Johnston J, Littau A, Roque R, et al. TACI-Ig neutralizes molecules critical for B cell development and autoimmune disease: impaired $\mathrm{B}$ cell maturation in mice lacking BLyS. Immunity. 2001;15(2):289-302.

9. Morel J, Roubille C, Planelles L, Rocha C, Fernandez L, Lukas $\mathrm{C}$, et al. Serum levels of tumour necrosis factor family members a proliferation-inducing ligand (APRIL) and B lymphocyte stimulator (BLyS) are inversely correlated in systemic lupus erythematosus. Ann Rheum Dis. 2009;68(6):997-1002.

10. Xin G, Shi W, Xu LX, Su Y, Yan LJ, Li KS. Serum BAFF is elevated in patients with IgA nephropathy and associated with clinical and histopathological features. J Nephrol. 2013;26(4):683-90.
11. Cheema GS, Roschke V, Hilbert DM, Stohl W. Elevated serum B lymphocyte stimulator levels in patients with systemic immunebased rheumatic diseases. Arthritis Rheum. 2001;44(6):1313-9.

12. Jonsson MV, Szodoray P, Jellestad S, Jonsson R, Skarstein K. Association between circulating levels of the novel TNF family members APRIL and BAFF and lymphoid organization in primary Sjogren's syndrome. J Clin Immunol. 2005;25(3):189-201.

13. Isenberg D, Gordon C, Licu D, Copt S, Rossi CP, Wofsy D. Efficacy and safety of atacicept for prevention of flares in patients with moderate-to-severe systemic lupus erythematosus (SLE): 52-week data (APRIL-SLE randomised trial). Ann Rheum Dis. 2015;74(11):2006-15.

14. Merrill JT, Wallace DJ, Wax S, Kao A, Fraser PA, Chang P, et al. Efficacy and safety of atacicept in patients with systemic lupus erythematosus: results of a twenty-four-week, multicenter, randomized, double-blind, placebo-controlled, parallel-arm, phase IIb study. Arthritis Rheumatol. 2018;70(2):266-76.

15. Munafo A, Priestley A, Nestorov I, Visich J, Rogge M. Safety, pharmacokinetics and pharmacodynamics of atacicept in healthy volunteers. Eur J Clin Pharmacol. 2007;63(7):647-56.

16. Dall'Era M, Chakravarty E, Wallace D, Genovese M, Weisman M, Kavanaugh A, et al. Reduced B lymphocyte and immunoglobulin levels after atacicept treatment in patients with systemic lupus erythematosus: results of a multicenter, phase $\mathrm{Ib}$, doubleblind, placebo-controlled, dose-escalating trial. Arthritis Rheum. 2007;56(12):4142-50.

17. Pena-Rossi C, Nasonov E, Stanislav M, Yakusevich V, Ershova $\mathrm{O}$, Lomareva N, et al. An exploratory dose-escalating study investigating the safety, tolerability, pharmacokinetics and pharmacodynamics of intravenous atacicept in patients with systemic lupus erythematosus. Lupus. 2009;18(6):547-55.

18. Nestorov I, Papasouliotis O, Pena Rossi C, Munafo A. Pharmacokinetics and immunoglobulin response of subcutaneous and intravenous atacicept in patients with systemic lupus erythematosus. J Pharm Sci. 2010;99(1):524-38.

19. Ansell SM, Witzig TE, Inwards DJ, Porrata LF, Ythier A, Ferrande $\mathrm{L}$, et al. Phase I clinical study of atacicept in patients with relapsed and refractory B-cell non-Hodgkin's lymphoma. Clin Cancer Res. 2008;14(4):1105-10.

20. Nestorov I, Munafo A, Papasouliotis O, Visich J. Pharmacokinetics and biological activity of atacicept in patients with rheumatoid arthritis. J Clin Pharmacol. 2008;48(4):406-17.

21. Rossi JF, Moreaux J, Hose D, Requirand G, Rose M, Rouille V, et al. Atacicept in relapsed/refractory multiple myeloma or active Waldenstrom's macroglobulinemia: a phase I study. Br J Cancer. 2009;101(7):1051-8.

22. Berelowitz K, Taubel J. Early phase Japanese bridging studies-global significance and CRO selection criteria. CRfocus. 2011;22(3):9-13.

23. Bhupathi C, Vajjha VH. Sample size recommendation for a bioequivalent study. Statistica. 2017;77:65-71.

24. European Medicines Agency. VICH GL 52: bioequivalence: blood level bioequivalence study. London: European Medicines Agency; 2015.

25. Hummel J, McKendrick S, Brindley C, French R. Exploratory assessment of dose proportionality: review of current approaches and proposal for a practical criterion. Pharm Stat. 2009;8(1):38-49.

26. Papasouliotis O, Yalkinoglu Ö, Golob M, Willen D, Girard P. Population pharmacokinetics of atacicept in systemic lupus erythematosus (SLE). Ann Rheum Dis. 2015;74(suppl 2):1077 (Abstract).

27. Gibiansky L, Gibiansky E, Kakkar T, Ma P. Approximations of the target-mediated drug disposition model and identifiability of model parameters. J Pharmacokinet Pharmacodyn. 2008;35(5):573-91. 
28. Statistics Bureau Japan. Ministry of Internal Affairs and Communications website: weight by age and sex (1900-2004). 2004. Available from: http://www.stat.go.jp/english/data/chouki/24.htm. Accessed 20 December 2016.

29. Ruston D, Hoare J, Henderson L, Gregory J, Bates CJ, Prentice A, et al. The national diet and nutrition survey: adults aged 19 to 64 years, vol. 4. Nutritional status (anthropometry and blood analytes), blood pressure and physical activity; 2004. London: The Stationery Office.

30. Bonilla FA. Pharmacokinetics of immunoglobulin administered via intravenous or subcutaneous routes. Immunol Allergy Clin North Am. 2008;28(4):803-19.

31. van Vollenhoven RF, Kinnman N, Vincent E, Wax S, Bathon J. Atacicept in patients with rheumatoid arthritis and an inadequate response to methotrexate: results of a phase II, randomized, placebo-controlled trial. Arthritis Rheum. 2011;63(7):1782-92.

32. van Vollenhoven RF, Wax S, Li Y, Tak PP. Safety and efficacy of atacicept in combination with rituximab for reducing the signs and symptoms of rheumatoid arthritis: a phase II, randomized, double-blind, placebo-controlled pilot trial. Arthritis Rheumatol. 2015;67(11):2828-36.
33. Dascalu C, Davidson A, Mackay MC, Furie R, Huang W, Aranow C. The effect of belimumab on peripheral blood cells in patients with systemic lupus erythematosus. Arthritis Rheumatol. 2015;67(suppl): 10 .

34. Stohl W, Hiepe F, Latinis KM, Thomas M, Scheinberg MA, Clarke A, et al. Belimumab reduces autoantibodies, normalizes low complement levels, and reduces select B cell populations in patients with systemic lupus erythematosus. Arthritis Rheum. 2012;64(7):2328-37.

35. Jacobi AM, Huang W, Wang T, Freimuth W, Sanz I, Furie R, et al. Effect of long-term belimumab treatment on B cells in systemic lupus erythematosus: extension of a phase II, doubleblind, placebo-controlled, dose-ranging study. Arthritis Rheum. 2010;62(1):201-10.

36. Tak PP, Thurlings RM, Rossier C, Nestorov I, Dimic A, Mircetic $\mathrm{V}$, et al. Atacicept in patients with rheumatoid arthritis: results of a multicenter, phase Ib, double-blind, placebo-controlled, doseescalating, single- and repeated-dose study. Arthritis Rheum. 2008;58(1):61-72. 